DOI 10.37882/2500-3682.2020.11.02

\title{
ВИДОВОЙ ДИНАМИЧЕСКИЙ СТЕРЕОТИП У ДЕТЕЙ ПЕРВОГО ГОДА ЖИЗНИ
}

\section{SPECIFIC DYNAMIC STEREOTYPE IN CHILDREN OF THE FIRST YEAR OF LIFE \\ S. Vishnev}

Summary: Dynamic stereotype (DS) is a complex multidimensional concept introduced by I.P. Pavlov in 1932 that denotes a system of conditioned and unconditioned reflexes, which manifests itself in the behavior of the organism, as a rule, in relation to a certain conditioned stimulus. Currently, DS is considered as a developed stable subjective reaction of the body to a conditioned stimulus.

The author's definition and practical application of included in DS the concept of species dynamic stereotype (SDS) allows us to pay attention to the formation of the main components of the DS, fixed in phylogeny, and therefore more important for ontogeny. In physiological terms, SDS as a phylogenetically fixed main part of DS maintains the resistance of reflex connections to phylogenetically significant unconditioned and conditioned stimuli. SDS is based on persistent connections between innate and conditioned reflexes and in turn is the basis for the formation of various motor stereotypes. SDS is connected to a number of similar concepts such as a species stereotype in ethology, a stereotyped form of behavior in zoopsychology, but it does not replace them. SDS as a complex of species stable reflex connections fixed in phylogeny in the structure of dynamic stereotype (DS) is an important link in the formation of motor activity and behavior in children of the first year of life. In practical meaning, the definition of SDS makes it easier to represent the formation and connections of general (large) and fine motor movements of a human as a biological species.

Our 20-year practical experience of observation and assistance in the formation of DS in more than 6000 children in the first year of life shows the methodological need to determine and take into account the properties of SDS in a particular child to control the dynamics and effectiveness of therapeutic and preventive measures. Based on the concept of SDS, the author has developed a number of practical techniques and recommendations for massage in pediatrics, the two patents for invention are prepared.

Keywords: dynamic stereotype (DS), species dynamic stereotype (SDS), reflex connection, optimal dynamic trajectory, kinematic chain.

\author{
Вишнев Сергей Юрьевич \\ ММЦ ООО МК «Здоровье Плюс», МЦ№2 г. Верхняя Пышма, \\ Свердловская область \\ vichserg@mail.ru
}

Аннотация: Динамический стереотип (ДС) - сложное многоплановое понятие, которое ввел И.П. Павлов в 1932 году, обозначает систему условных и безусловных рефлексов, которая проявляется в поведении организма, как правило по отношению к определенному условному раздражителю. В настоящее время ДС трактуется как выработанная устойчивая субъективная реакция организма на условный раздражитель.

Определение автором включаемого в ДС, понятия ВДС и его практическое применение позволяет обратить внимание на формирование основных, закрепленных в филогенезе, а значит наиболее важных для онтогенеза, компонентах ДС. В физиологическом значении, ВДС, как филогенетически закрепленная, основная часть ДС, поддерживает устойчивость рефлекторных связей к филогенетически значимым безусловным и условным раздражителям. ВДС базируется на стойких связях врожденных и условных рефлекСов и, в Свою очередь, является основой при формировании двигательных стереотипов разного порядка и уровня организации. ВДС взаимосвязан, но не подменяет ряд сходных по смыслу понятий, таких как видовой стереотип в этологии, стереотипная форма поведения в зоопсихологии. Видовой динамический стереотип (ВДС) как комплекс закрепленных в филогенезе видовых устойчивых рефлекторных связей в структуре динамического стереотипа (ДС) является важным звеном в формировании двигательной активности и поведения у детей первого года жизни. В прикладном значении, определение ВДС позволяет легче представлять образование и связи обще (крупно) и мелко моторных движений человека, как биологического вида.

Наш 20-летний практический опыт наблюдений и помощи в формировании ДС у более чем у 6000 детей первого года жизни, показывает методическую необходимость определять и учитывать свойства ВДС у конкретного ребенка для контроля за динамикой и эффективностью лечебных и профилактических мероприятий. На основе представлений о ВДС, автором разработаны ряд практических методик и рекомендации по массажу в педиатрии, подготовлены два патента на изобретения.

Ключевые слова: динамический стереотип (ДС), видовой динамический стереотип (ВДС), рефлекторная связь, оптимальная динамическая траектория, кинематическая цепь.

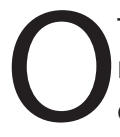

ткрытое и обстоятельно изученное И.П. Павловым и его учениками, явление динамического стереотипа (ДС) [13], в настоящее время широко используется и продолжает исследоваться в философии, социологии, биологии, медицине, физиологии, психологии, биомеханике и ряде других дисциплин. Выявленная школой И.П. Павлова, динамическая устойчивость связей условных и безусловных рефлексов, применяе- мая в конкретном случае организмом к условному, а не воздействующему раздражителю, - в настоящее время получила более широкую трактовку, как выработанная устойчивая субъективная реакция (концепт, комплекс, действие, акт, координация, генерация, рациональность и т.п.) системы или совокупности рефлекторных связей на условный раздражитель [8]. Указывая его (ДС) строго индивидуальное значение для самого субъекта, также, 
в настоящее время, по принципу адекватности формирования и использования самим субъектом, принято различать оптимальный двигательный стереотип (ОДС) и неоптимальный двигательный стереотип (НДС). Как автор статьи, я прошу обратить внимание на индивидуальность, как свойство, присущее формированию и выражению явления ДС [2]. И тогда возникает вопрос. Откуда появится это свойство, без логического противопоставления индивидуальности самому виду (Homo sapiens) и общебиологического принципа неотделимости вида от среды существования? Только лишь запоминание положительной для индивида значимости (впервые сформулированной Л.С. Выготским), возникающей в результате реализации «случайных проб и ошибок», без наличия «врожденного эталона» будет явно недостаточно [4]. Поэтому, считаю необходимым и целесообразным, различение в составе явления ДС видовой составляющей, как идеальной структуры, и предлагаю обозначить ее как видовой двигательный стереотип (ВДС). В отличие от свойств ОДС, свойства ВДС определяют способы образования, долговременного хранения и сохранения связей условных и безусловных рефлексов относительно всего времени существования рассматриваемого биологического вида в его среде обитания. В свою очередь, отдельно рассматриваемый индивид (субъект) является носителем этих свойств ВДС, что делает сам ВДС не абстрактным, а физическим понятием.

Траектории движения, основные опорные позы (положения тела) и основные движения в видовой продуктивной деятельности объединены в ВДС между собой, подчинены логике видовой реализации законов классической механики в среде существования рассматриваемого вида, мало зависят от конституции, пола, возраста, других переменных и особенностей. Связи рефлексов базируются на врожденных рефлексах и адаптируются самим субъектом к среде существования, служат эталоном при формировании двигательных стереотипов разного порядка и уровня организации.
В этой статье предлагается определение ВДС как комплекса наиболее устойчивых, филогенетически закрепленных рефлекторных связей, обусловленный процессами видообразования. В физиологическом значении, ВДС, как филогенетически закрепленная часть ДС, поддерживает устойчивость рефлекторных связей к филогенетически значимому условному раздражителю.

В концептуальном значении, ВДС можно определить как структурную часть ДС с характерной для конкретного вида динамической устойчивостью связей условных и безусловных рефлексов, применяемых в конкретном случае организмом к условному раздражителю. По сути, ВДС выступает в роли филогенетически закрепленного «маркера» устойчивости рефлекторных связей. По определению, ВДС взаимосвязан, но не подменяет ряд смежных с ним понятий, например, видовой стереотип поведения в этологии'. Также, очевидно соотношение понятия ВДС с понятием, принятым в адаптивной зоопсихологии - стереотипной формы поведения, где реакция на эффект (результат) поведенческой активности противопоставлена форме движения. Или, взаимосвязь с понятием в когнитивном значении принципа решения адаптивных задач, где этот принцип - сложный процесс согласования и управления элементарными навыками. Определяя содержание ВДС, руководствуемся правилом, сформулированным Л. Морганом (1894 г.), означающим что, не следует привлекать к объяснению поведения животных более высоких психических категорий, чем необходимо. Во-первых, ВДС не имеет прямого отношения к устойчивости и ряду других характеристик содержания безусловного и условного рефлекса, для этого существует другой механизм реализации. Вовторых, ВДС не является «эталонной» составляющей форм поведения, в том числе и инстинктов, поскольку не может быть оформленным звеном или элементом любого из них. В-третьих, ВДС определяется необходимостью и целесообразностью решения адаптивных филогенетически значимых задач, применительно к определенным ситуациям, проявляющихся в среде, где применение

В этологии (К. Лоренц, Н. Тинберген, К.Фриш, М.Л. Бутковская, Ю.М. Плюснин и др. авторы [3]) именно видовые стереотипы поведения, в основе своей наследуемые, представляют собой главный предмет исследования. Их возникновение в процессе эволюции (по Н. Тинбергену) легко объяснимо: генетически запрограммированные стереотипы поведения позволяют осуществить адаптацию быстро, с минимальным расходом энергии. Поведение - (по Л.С. Выготскому) процесс взаимодействия между организмом и средой. Видовой стереотип поведения - (этологическое определение) это приспособление к наиболее общим и постоянным условиям среды. Именно постоянство условий среды является фактором, который ведет к закреплению отвечающей ему формы поведения. Стереотипная поведенческая реакция может формироваться и по отношению к меняющимся условиям среды, если эти изменения повторяются закономерно. Полной идентичности условий, абсолютной их повторяемости из года в год в природе не существует. Поэтому видовые стереотипы поведения никогда не состоят только из наследственно закрепленных реакций (инстинктов). Врожденные реакции - это основа сложного поведенческого акта, на который накладывается научение. Видовые стереотипы поведения устойчивы, а поведение на основе научения отличается пластичностью. Генетически фиксированы и передаются по наследству не отдельные готовые движения или их сочетания, а нормы реагирования, в пределах которых формируются двигательные реакции в онтогенезе. Способность к научению, к установлению новых ассоциаций, тоже видоспецифична. Важно, что инстинктивное поведение обладает вариабельностью, а выученное поведение не может выйти за границы, определенные видовой принадлежностью. 
других средств решения оказывается не эффективным. То есть, для решения ситуативной задачи безрезультатно использовать одиночный рефлекс или случайную комбинацию рефлексов, либо излишне вырабатывать поведенческую стратегию решения данной задачи в изменяющихся условиях среды.

В качестве весомого аргумента в пользу сказанного, предлагаю рассмотреть таблицу связей некоторых безусловных и условных моторных рефлексов ребенка первого года жизни, раскрывающую последовательность формирования обще моторных (крупно моторных) движений ребенка (см. рис. 1).
Все сокращения в рисунке соответствуют сокращенным обозначениям моторных рефлексов ребенка первого года жизни. Более подробное описание содержания этих рефлексов можно посмотреть в различных источниках (например, в работах А.Ю. Ратнера, Н.П. Шабалова, В.Л. Страковской $[12,14])$. Обращаю внимание на то, что выведенная в таблице устойчивая последовательность некоторых связей рефлексов с движениями достаточно просто определяется в лонгитюдном наблюдении за формированием обще моторных движений детей возраста до трех лет. Как следует из наблюдений, цепи рефлексов и связанных с ними движений, имеют различные варианты построения (разветвленные, цикличные, ступенчатые, параллельные, пересекающиеся, последовательные и др.).

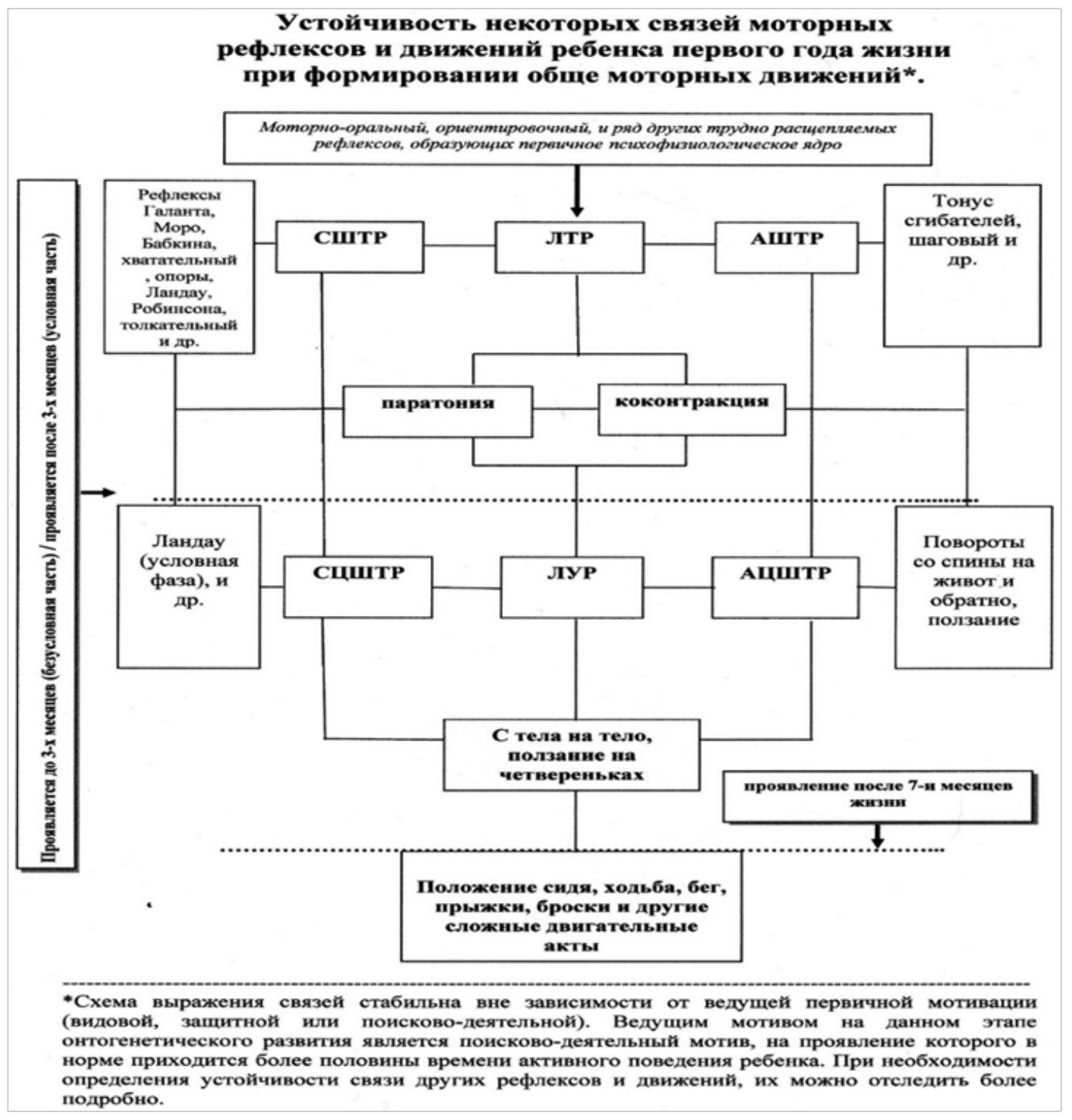

Рис. 1. 
Живой организм никогда не находится в бездеятельном состоянии относительно среды, при этом, его активность определяется потенциальными возможностями тела и его реактивным состоянием. Физическая и функциональная незрелость различных систем организма ребенка определяет его качественное отличие характеристики активности в сравнении с активностью взрослого организма. Как и ДС, ВДС ребенка по сравнению со взрослым, можно охарактеризовать более выраженной инвариативностью проявлений, зависящей от специфики решения адаптационных задач, связанных с применением ДС самим ребенком. В ВДС ребенка сохраняется динамическая устойчивость связей рефлексов по отношению к условному раздражителю, где области определения задач видовой реализации в среде остаются постоянными. То есть, для ВДС ребенка, как активного процесса, выразительны общие со взрослым условия активности организма, имеющие значение вне зависимости от состояния зрелости. Общим условием потенциальной активности организма выступает удовлетворение самим организмом физических и физиологических потребностей существования данного вида в среде. Для этого, как минимум, требуется устойчивая совокупность когнитивных, оперативных и продуктивных действий. Поэтому, как автор, считаю не совсем корректной точку зрения о связи ДС только с оперативной составляющей, представленной в психомоторной организации человека (Е.П. Ильин [7]). Если для продуктивных действий наличие предшествующих врожденных рефлексов и их связей различить достаточно сложно, то для когнитивных действий имеется вполне определенное основание (связи поискового, ориентировочного и др. врожденных рефлексов). Тем не менее, для описания целесообразности определенной фиксации связей рефлексов по отношению к условному раздражителю, воспользуемся несколько иным, физическим основанием [15].

Из курса классической механики известно, что гравитационная сила действует на все без исключения земные объекты, в том числе и живые организмы. Гравитационная нагрузка активизирует системы живого организма и стимулирует его развитие, является регулятором кинематических цепей его опорно-двигательной системы (В.А. Мицкевич [9]). Из школьного курса классической механики следует, что при рассмотрении физической модели (абсолютно твердого или абсолютно упругого) тела для определения его центра тяжести необходимо приложить вектор силы тяжести к центру массы рассматриваемой модели тела. Обращаю ваше внимание к тому, что центр массы тела ребенка располагается значительно выше (в проекции мечевидного отростка или несколько выше), по сравнению с взрослым человеком (в проекции 1-2 поясничного позвонка, в движении допускается ниже). Отсюда следует, что центр массы тела в физической модели живого существа не только меняет свое координатное положение в процессе роста организма, но и постоянно колеблется и перемещается по определенным траекториям в результате внутренних движений самого живого организма.

Это, в свою очередь, влечет за собой изменение кинематических цепей, зависящих от пространственного положения центра тяжести. Кроме того, все живые существа располагаются и перемещаются в физическом пространстве не хаотично, и не постоянно вращаясь вокруг собственного центра массы, или перекатываясь как колобок, а сохраняют определенное относительное положение тела в физическом пространстве. Так, для всех живых организмов, имеющих кишечную трубку важно располагать ее ротовой конец в направлении к конкретному объекту или точке приближения в среде, при этом, направлять максимальный поперечник входной части (полости рта) строго по этой линии (с допустимым отклонением просвета, или поперечника входной части, менее трех градусов). Во время движения относительное положение этой линии и центра массы тела «строго фиксировано» и контролируется самим живым организмом. Человек не является исключением из этого правила, что выражено в «аллегорической» поговорке «Никто, никого, ни в одной религии, не учит молиться носом кверху». Целесообразность и образ позы и движения, молящегося во время молитвы (для того хотя бы, чтобы молящийся не остался голодным), прекрасно иллюстрируют это правило. До пяти месяцев жизни (чаще до четырех) здоровый ребенок стремится «запрокидывать» голову, стремясь удержать «линию движения рта» относительно туловища в положении, характерном для водных существ. И, не удивительно, ведь весь его предшествующий «опыт» внутриутробных движений приобретался в «водной» среде, где для большинства водных существ характерно располагать «линию движения рта» строго соосно хорде, или продольной осевой линии (длиннику) туловища. И лишь затем ребенок переучивается «контролировать» положение этой линии относительно направления движения «на суше». По некоторым статистическим данным, только около трети детей делают это (переучиваются) легко и мало заметно для окружающих. Также, нетрудно сделать вывод о значимости лабиринтно-тонического (ЛТР) и лабиринтно-установочного рефлексов (ЛУР) и их связей для понимания этого процесса.

Традиционно, характеристику движений человеческого организма в физическом пространстве принято рассматривать в логике связей и построения процесса в статике, в стато-кинетике и динамике. При рассмотрении связей кинематических цепей, для живых существ, обладающих локомоцией, также важен суммарный вектор направления движения, проявляющийся в ходе все- 
го онтогенеза рассматриваемого живого существа. Данный вектор имеет выражение одновременно для всех случаев выполнения движений в поступательном направлении. Согласно традиции, рассмотрение движения следует начинать от исходного положения - статической позы. В классической механике статическое положение физического объекта (например, материальной точки) принято рассматривать лишь как частный случай его движения с нулевым ускорением, и описание движений, наоборот, следует из динамической характеристики. Поэтому, вопрос о движениях человека и формировании ДС ребенка рассматривается иначе, - «Как от движений в лежачем положении перейти к движениям в прямохождении»? В этом ключе становится очевидной неразделимость поз, а также, ведущая роль динамических связей по отношению к статическим позам как в самом движении, так и в структурах управления движением в целом. Нет смысла раскрывать весь материал, касающийся классической механики в этой статье, приведу лишь наиболее значимые относительно содержания ВДС положения и теоремы. Это основные положения кинематики, понятия системы отсчета, первый и второй законы Ньютона, теоремы Грасгофа, Кориолиса, первая и вторая теоремы Резаля [6]. Из основных положений кинематики следует другой способ описания возможных обще моторных движений и связанных с ними поз. Обще моторные движения по твердой поверхности возможны: с максимальным или значительным использованием опоры на туловище; в комбинации с туловищем или изолированным использованием конечностей для опоры; с частичным использованием дистальной части туловища и применением только нижних конечностей для опоры; с использованием опоры только на нижние конечности. В первом случае возможны только барахтанье, перевороты, движение перекатом и «змейкой». Во втором ползание (лазание по плоскости) в положении низких, средних и высоких четверенек. В третьем - движения в положении «сидя», привставания на колени, или стояние с опорой на руки или туловище. В четвертом - свободное стояние и приседание, ходьба, бег, прыжки и броски предмета по баллистической траектории. Считаю, что дальнейшие комментарии в необходимости изучения и применения в теории и практике физического принципа - излишним. Описание поз, статических, кинетостатических и статокинетических связей, общие выводы о построении мелкой моторики [5], из сказанного можно вывести самостоятельно.

В характеристике «сухопутной» среды и ее значимости для опоры во время движения обозначу лишь несколько моментов. В плотной «водной» среде имеется возможность «опираться» на нее всем телом (то есть, любой его частью), кроме того, из-за этой плотности сами движения приобретают реактивный, или вол- нообразный вид, с ведущей ролью в качестве привода «хвостовой части» - плавника или нижних конечностей. Верхние конечности или плавники играют роль стабилизаторов траектории движения. Поэтому и рука человека обычно «развивается» в обще моторном смысле, с некоторым «запаздыванием» и дифференцированием функций ближе к началу ходьбы. В «сухопутной» среде имеет значение траектория перемещения, с точками касания, расположенными впереди двигающегося по ходу перемещения. Поверхность, по которой осуществляется перемещение, рассматривается как препятствие. Этим препятствием может быть плоскость (протяженная, ограниченная, горизонтальная, с уклоном с определенно значимым угловым значением для способа движения, вертикальная), уступ, выступ, яма и т.п. Препятствие может быть различно расположено относительно, и под различным углом, к направлению движения (продольно, поперечно, касательно, по ходу, параллельно). В шутку отмечу, что «Слава богу, летать не учимся!», и всерьез также, предлагаю рассмотреть «алгоритм» оптимального преодоления препятствия самостоятельно. В механическом смысле, эта характеристика выполняется через определение степеней свободы движения и приложение к системе необходимого числа дополнительных опорных точек.

Определяя ДС, в том числе и ВДС, как психомоторный акт, и различая его психологическую составляющую, обозначим ее как психологически структурированную экономию потребностей в двигательной активности и действиях (Ж. Пиаже, Б. Инхельдер [11]). Формирование ДС зависит от эффективности осознавания своих потребностей самим субъектом [10]. Первичные мотивы (защитный, видовой и поисково-деятельный), в свою очередь, служат психологическими регуляторами степени удовлетворенности потребностей субъекта. Обусловленная мотивация не только экономит ресурсы организма, но и поддерживает цикличность взаимодействий по отношению к определенно сгруппированным и значимым факторам среды. Цикличность активности организма, также, в какой-то степени, может быть следствием этого эффекта. ДС может быть проявлен под действием любого из первичных мотивов, но в норме, и наиболее эффективно формируется и реализуется в поисково-деятельном мотиве (более половины активного времени поведения субъекта). Обращаю внимание на то, что в сенсомоторном периоде любой мотив может быть проявлен ребенком только в отношении контакта, либо игнорирования конкретно проявленного для ребенка фактора. Ребенок не отделяет, или мало различает себя с предметной и физической реальностью. Поэтому, любой фактор имеет значение только в случае его выделения самим ребенком. Также, среди значимых для конструкции схем сенсомоторной реальности и выражения пер- 
вичных мотивов, следует обозначить эффект обратимости Дж. Келли, - одновременно связанный с процессами памяти, вниманием, осознанием, и выражением эмоций 2.

Сталкиваясь и оказывая влияние в занятиях, занимаясь практическим формированием ДС у детей первого года жизни, предлагаю учитывать свойства ВДС в своей практике. Суть метода определения ВДС заключается в использовании элементов целенаправленного обстоятельного неврологического и психологического осмотра как во время первого занятия, так и в ходе последующих занятий. То есть, оцениваем качество исполнения в движениях ребенка, как самих рефлексов, так и их связей. При выполнении отдельных движений или упражнений следует отслеживать и корректировать точность их выполнения по оптимальным динамическим траекториям [1]. При любом изменении скорости или направления выполняемого движения (упражнения) отслеживаем и корректируем качество «группировки вперед», по направлению движения. При выполнении «группировки вперед» используется связь ЛТР и ЛУР строго по траектории движения. Сначала одновременно и симметрично включаются в работу сгибатели шеи (кивательные мышцы), затем растягиваются разгибатели, без участков сжатия в шейном и верхне-грудном отделах позвоночника, при этом, затылочный выступ перемещается вперед, за плоскость лопаток (приложенную к внутренней плоскости обеих лопаток). В организационном плане, отслеживаем триаду: равновесие, координированность и порядок выполнения элементов движений. Любой ребенок старается «переложить» эту триаду на взрослого, оставляя себе только свои собственные желания. Качество динамического и статического равновесия ребенка отслеживаем и корректируем в соответствии с возрастной нормой и фактическим умением ребенка. В ходе выполнения упражнений не только сами исправляем ошибки координированности движений ребенка, но и стараемся вовлечь самого ребенка в процесс исправления ошибок. Устанавливая порядок выполнения элементов движений и действий в упражнении, сначала определяем оптимальную, возможно допустимые и обратимые траектории движений ребенка и его возможные варианты выполнения действий. При освоении опорных и физиологических поз, обязательно включать в подборку динамические упражнения, направленные на сохранение этих поз в движении. При безрезультатных 5-7-и попытках ребенка справиться самостоятельно, следует упростить выполняемую им задачу. Вспомогательные поддержки обычно стремимся выполнять из-под центра массы, либо, контролируя его (центр массы) перемещение, то есть необходимо избегать выполнения работы по механическому принципу «устойчивости вагонетки», строго дозируя нагрузку и ускорение. Общим признаком утомления и необходимости остановки занятия служит «обмякание плеч» и «не правильная группировка» при выполнении движения ребенком. При этом, к повторению занятия можно вернуться, не ранее, чем через два часа. Обычно, утомление при выполнении упражнений на равновесие, координированность и порядок выполнения движений у детей возраста до года наступает в течение 5-15-ти минут. Утомление при выполнении тех же упражнений в статическом положении наступает в течение 1-3-х минут. Ожидание принятия решения ребенком о самостоятельном движении или действии от 15-ти секунд до 2-х минут, в зависимости от ситуативной задачи. Общее рекомендуемое основное время занятия, индивидуальное, 5-20 минут. Рекомендательный список можно продолжить далее, но необходимо учитывать, что это ознакомительная статья о формировании ДС у детей первого года жизни, а не методическое руководство.

Основными выводами из представленного материала в статье считаю, что ВДС является важным звеном в формировании и построении движений и действий. ВДС базируется на стойких связях врожденных и условных рефлексов и, в свою очередь, является основой при формировании двигательных стереотипов разного порядка и уровня организации. Формирование динамического стереотипа, выполняемое с применением ВДС происходит результативнее, быстрее и с лучшим качеством, нежели спонтанно, на основе случайных проб и ошибок. Сравнить значение ВДС можно с письмом по прописям при освоении письменной речи ребенком, где дальнейшее изменение почерка будет происходить индивидуально. Как и любая идеальная структура, ВДС преобразуется в индивидуальную форму ДС в зависимости от условий и активности самого субъекта, то есть ребенка.

2 Действие эффекта, вероятно, основывается на врожденной способности к счету в пределах первых двух десятков, активно проявляемой ребенком старше полутора лет. Эмоциональное выражение эффекта Дж. Келли состоит в следующем: столкнувшись впервые с незнакомым раздражителем, субъект реагирует панически, или с высшей тревожностью. При столкновении до 5 раз с этим раздражителем, сохраняет высокий уровень тревожности. При столкновении от 5 до 10 раз, выражает удовольствие при возможности проигнорировать факт столкновения. При столкновении от 11 до 15 раз в равной степени проявляет активность и игнорирует раздражитель, выражая разнообразные эмоции. При столкновении от 15 до 20 раз полностью регулирует активность в отношении раздражителя, выражая разнообразные эмоции. И, при столкновении более 20 раз, и, если способен организовать свои движения и действия в обратном порядке, - значительно перестает эмоционально реагировать, многократно (около 100 раз) и качественно усиливает внимание, активно и долговременно запоминает свои действия. В столь подробном описании, эффект Дж. Келли показан из-за его практического значения в формировании ДС. 


\section{ЛИТЕРАТУРА}

1. Акопян Л.В., Павлухина Н.П., Яшина Т.Н., Массаж в педиатрии. Теория и практика: Учебное пособие. - СПб.: Наука и Техника, 2019. - 528 с., С. $37-44$, 518-521

2. Аникина Т.А., Крылова А.В., Физиологические основы высшей нервной деятельности детей и подростков: уч.-метод. пособие - Казань: Казанский федеральный университет, Ин-т физической культуры, спорта и восстановительной медицины, 2014, C. 13-24. https://kpfu.ru/

3. Бутковская М.Л., Плюснин Ю.М., Принципы организации пространственного поведения у человека и высших приматов (сравнительный анализ) // Современная антропология, генетика и проблема рас у человека / ред. И.М. Золотарева, Г.А. Аксякова. - М : Институт этнологии и антропологии им. Н.Н. Миклухо-Маклая РАН, 1995, С. 91-143.

4. Веккер Л.М., Психика и реальность: Единая теория психических процессов. - М.: Смысл; Per Se, 2000. - 685 с., С. 54-75

5. Вишнев. С.Ю., Патент РФ №2531724, 2014, материалы реферата к патенту.

6. Голубева О.В., Петрова Н.П., Механика Галилея-Ньютона и Энштейна, Институт проблем механики РАН, УрГПУ, Москва - Екатеринбург, $1994 .$, С. 3-68

7. Ильин Е.П., Психомоторная организация человека. Учебник для вузов, - СПб.: Питер, 2003. - 384 с. (Серия «Учебник нового века»)., С. 8-23, 42-123

8. Курпатов А.В., Аверьянов Г.Г., Руководство по системной поведенческой психотерапии. Динамический стереотип как концепт., https://iknigi.net/avtorandrey-kurpatov/51876-rukovodstvo-po-sistemnoy-povedenchenskoy-psihoterapii-andrey-kurpatov/read/page-1.html, C.5-7

9. Мицкевич В.А., Ортопедия первых шагов. М.: БИНОМ. Лаборатория знаний, 2013. - 359 с., С. 5-91

10. Новоселова С.Л., Развитие интеллектуальной основы деятельности приматов. -Москва: Изд-во Московского психолого-социального ин-та; Воронеж: НПО «МОДЭК», 2010. -2-е изд., - 304 с.

11. Пиаже Ж. Инхельдер Б., Психология ребенка: Науч. ред. пер. С.Л. Соловьева. - 18-е изд. - СПб.: Питер. 2003. - 160 с. (Серия «Концентрированная психология»), (. 7-39

12. Страковская В.Л., Лечебная физкультура в реабилитации больных и детей группы риска 1-го года жизни». - М.: Медицина, 1991, 160 с.

13. Хананашвили М.М., Динамический стереотип // Большая медицинская энциклопедия / под ред. Б.В. Петровского, 3-е издание, т. 3, - М. - Л., 1951., httрs://бмэ.оргіindex.php/ДИНАМИЧЕСКИЙ СТЕРЕОТИП

14. Шабалов Н.П., Неонатология /Учебное пособие: в 2 т. - 3-е изд., испр. и доп. - М.: МЕД пресс-информ, 2004. - 640 с., том 1, С. 178-221

15. Яворский Б.М., Детлаф А.А. Справочник по физике. - 2-е изд., перераб. - М.: Наука, Главная редакция физико-математической литературы, 1985, 512 c., C. 11-53, 404-406

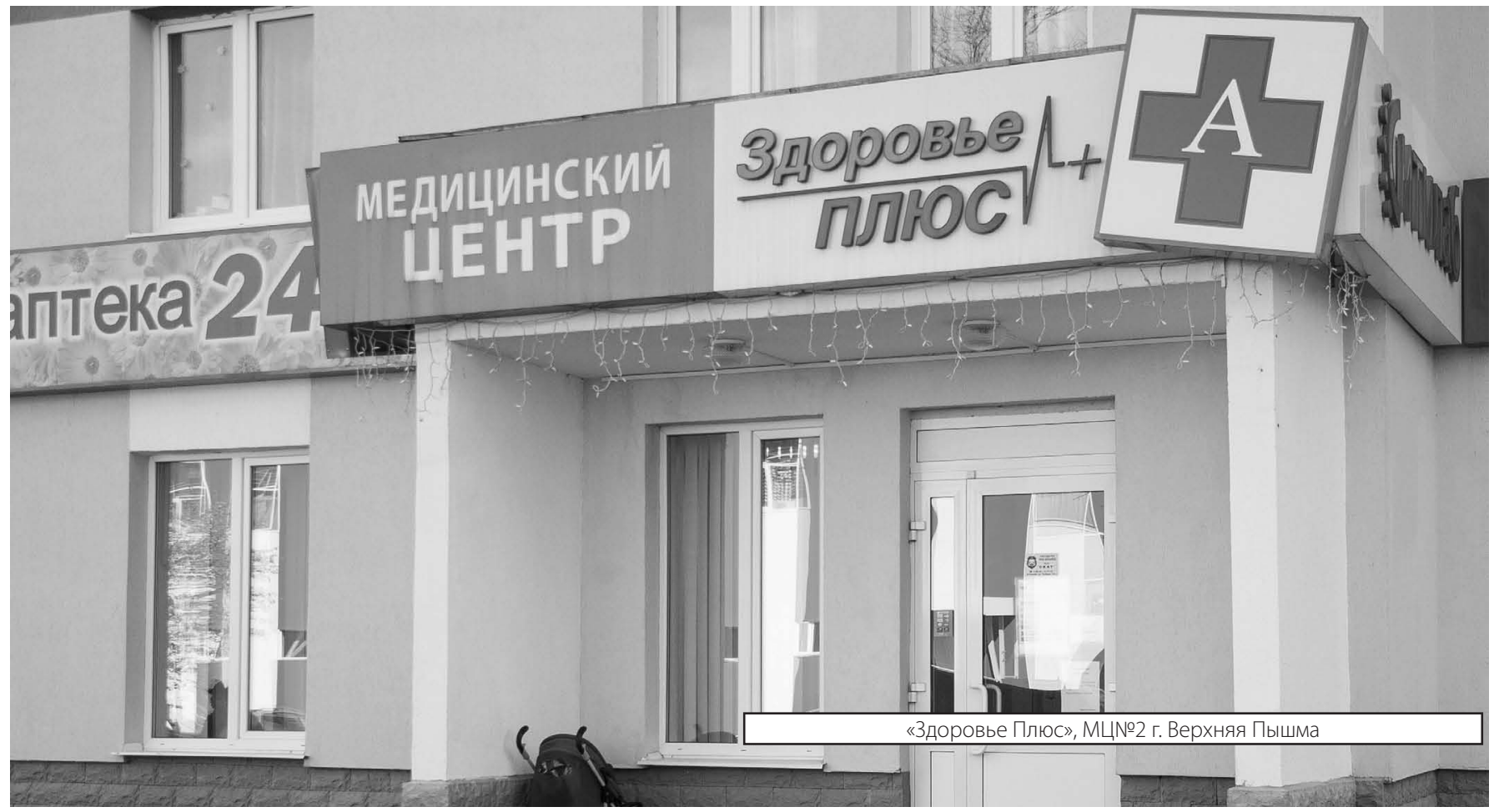

